https://doi.org/10.22319/rmcp.v12i1.5392

Nota de investigación

\title{
Nivel de infestación de Rhipicephalus microplus y su asociación con factores climatológicos y la ganancia de peso en bovinos Bos taurus $\mathbf{x}$ Bos indicus
}

\author{
Roberto Omar Castañeda Arriola ${ }^{\text {a }}$ \\ Jesús Antonio Álvarez Martínez ${ }^{\text {b }}$ \\ Carmen Rojas Martínez ${ }^{b}$ \\ José Juan Lira Amaya ${ }^{b}$ \\ Ángel Ríos Utrera ${ }^{a *}$ \\ Francisco Martínez Ibáñez ${ }^{\mathrm{c}}$
}

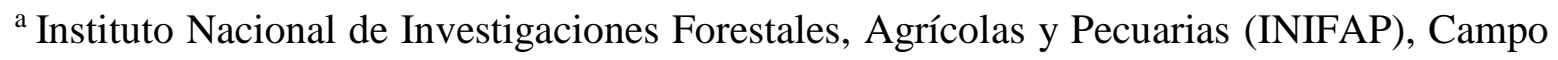
Experimental La Posta, kilómetro 22.5 carretera federal Veracruz-Córdoba, Paso del Toro, Medellín, Veracruz, México.

${ }^{\mathrm{b}}$ Instituto Nacional de Investigaciones Forestales, Agrícolas y Pecuarias (INIFAP), CENIDSAI, Morelos, México.

${ }^{\text {c }}$ Servicios de Sanidad, Inocuidad y Calidad Agroalimentaria (SENASICA), Ciudad de México, México.

*Autor de correspondencia: rios.angel@inifap.gob.mx

\section{Resumen:}

El objetivo fue evaluar el grado de infestación natural por Rhipicephalus microplus, así como determinar su correlación con factores climatológicos y su efecto sobre la ganancia de peso en bovinos doble propósito. Se utilizaron 31 bovinos cruzados, Bos taurus x Bos indicus, de ambos sexos, con una edad promedio de 307 días. El conteo de garrapatas semi-repletas (4.5 a $8.0 \mathrm{~mm}$ de diámetro) y el pesaje de los animales se realizaron cada 28 días. El conteo de 
garrapatas se realizó desde la cabeza hasta la base de la cola, abarcando miembros anteriores y posteriores, así como la región ventral de los animales. Las variables de respuesta estudiadas fueron número de garrapatas semi-repletas y ganancia de peso promedio del animal. En julio el número promedio de garrapatas por animal fue mayor $(P<0.05)$ que en el resto de los meses del año. Por el contrario, el sexo y el grupo racial del animal no afectaron $(P>0.05)$ el número de garrapatas. La ganancia de peso disminuyó $34 \mathrm{~g}(P<0.05)$ por cada garrapata semi-repleta por periodo. El número de garrapatas tuvo una correlación baja $(P<0.01)$ con la temperatura ambiental y humedad relativa; ganancia de peso promedio correlacionó negativa y moderadamente con el número de garrapatas $(-0.67 ; P<0.01)$. Los bovinos con un nivel de infestación alto (61 garrapatas o más) tuvieron una ganancia de peso promedio menor ( $P=0.001)$ que aquellos con nivel de infestación medio (31 a 60 garrapatas) y bajo (0 a 30 garrapatas). La infestación de bovinos de doble propósito con Boophilus microplus requiere un control más estricto en los meses de mayor temperatura ambiental (abril a julio).

Palabras clave: Garrapatas, Nivel de infestación, Ganancia de peso, Temperatura ambiental, Regresión lineal.

Recibido: 22/05/2019

Aceptado: 03/06/2020

El vector Rhipicephalus microplus representa un gran riesgo para el sector ganadero, debido a que afecta la producción de carne y leche. Además, es importante destacar que representa una barrera comercial y una amenaza para la venta de ganado en pie a los Estados Unidos de América, actividad que genera 700 millones de dólares al año ${ }^{(1)}$. La estrategia empleada para el control de la garrapata se ha basado en la aplicación de compuestos químicos, no obstante, estos pueden resultar tóxicos y costosos. Aunado a esto, hay que considerar que la generación de resistencia de las garrapatas ha propiciado replanteamientos sobre este método ${ }^{(2)}$. El manejo integral de plagas se considera la mejor opción en el control de las garrapatas, pero para lograrlo es necesario tener profundos conocimientos de las interacciones entre ambiente, hospedero y parásito. Mediante esta forma de control se logra tener poblaciones de garrapatas en cantidades bajas, para no poner en riesgo la salud de los bovinos, pero que a su vez sean suficientes para infectarlos con patógenos hemotrópicos a temprana edad; de esta forma, podrán generar inmunidad contra los mismos, logrando por consiguiente una estabilidad enzoótica $^{(3)}$.

El conocimiento de la variación poblacional a lo largo del año, así como de la influencia del clima y del manejo sobre la garrapata, son fundamentales para establecer un adecuado 
control. Por lo anterior, el objetivo del presente trabajo fue evaluar el grado de infestación natural por Rhipicephalus microplus, así como determinar su correlación con factores climatológicos y su efecto sobre la ganancia de peso en bovinos doble propósito.

El estudio se desarrolló en el campo experimental La Posta, perteneciente al Instituto Nacional de Investigaciones Forestales, Agrícolas y Pecuarias (INIFAP), localizado en el kilómetro 22.5 de la carretera federal Veracruz-Córdoba, en Paso del Toro, Medellín, Veracruz, México, a los $19^{\circ} 00^{\prime} 49^{\prime \prime} \mathrm{N}$ y $96^{\circ} 10^{\prime} \mathrm{O}$, a una altura de $12 \mathrm{~m}$ sobre el nivel del mar, con clima subhúmedo (Aw1), temperaturas máxima, media y mínima de 35.3, 25.0 y $15.0^{\circ} \mathrm{C}$, respectivamente, precipitación anual de $1,641 \mathrm{~mm}$ y humedad relativa de $74.4 \%$.

Se utilizaron 31 bovinos (13 vaquillas y 18 toretes) cruzados Bos taurus x Bos indicus, 11/16 Holstein x 5/16 Cebú (3.2\%), 11/16 Suizo Pardo x 5/16 Cebú (3.2\%), 3/4 Holstein x 1/4 Cebú (16.1\%), 3/4 Suizo Pardo x 1/4 Cebú (25.8\%), 5/8 Holstein x 3/8 Cebú (25.8\%), 5/8 Suizo Pardo x 3/8 Cebú (6.5\%), cruzas indefinidas de Holstein x Cebú (16.1\%), y cruzas indefinidas de Suizo Pardo x Cebú (3.2\%). La edad promedio de los animales al inicio del experimento fue de 307 días. Los toretes y las vaquillas se mantuvieron separados, en pastoreo rotacional en potreros contiguos establecidos con Tanzania (Megatyrsus maximus), Señal (Urocholoa decumbens), Pangola (Digitaria decumbes) y Mombaza (Megatyrsus maximus). Se ofrecieron sales minerales y agua a libertad todo el año; además, en la época de sequía (diciembre a mayo) se proporcionó ensilaje de sorgo forrajero (Sorghum vulgare) ad libitum. La vacunación contra derriengue (rabia paralítica) se realizó anualmente, en agosto. Los animales se desparasitaron contra nematodos gastroentéricos cada seis meses y se vacunaron contra clostridiasis en marzo y septiembre. Los bovinos se suplementaron con un alimento concentrado (18\% de proteína cruda y $70 \%$ de nutrientes digestibles totales) a razón de $1 \mathrm{~kg}$ por animal por día.

Los animales fueron inspeccionados con la finalidad de registrar la dinámica poblacional del vector Rhipicephalus microplus mediante el conteo de garrapatas semi-repletas (4.5 a 8.0 $\mathrm{mm}$ de diámetro), alojadas sobre ellos; los muestreos de garrapatas se realizaron de agosto del 2014 a octubre del 2015. La colecta y el conteo de garrapatas los realizó una misma persona, en un corral de manejo techado, iniciando a las 08:00 h. La periodicidad de los conteos fue cada 28 días, los cuales se realizaron desde la cabeza hasta la base de la cola, abarcando miembros anteriores y posteriores, así como la región ventral de los animales ${ }^{(4)}$. En cada muestreo se obtuvo el peso corporal de los animales con una báscula electrónica. Durante el estudio, no se aplicó ningún garrapaticida a los animales. En cada muestreo, las garrapatas se colectaron en una solución de alcohol al 70\%; posteriormente se llevaron al laboratorio de sanidad animal del campo experimental La Posta, para corroborar su clasificación taxonómica de acuerdo como lo describen Rodríguez-Vivas et al ${ }^{(5)}$. Los datos de temperatura ambiental y humedad relativa se obtuvieron de la estación climatológica del campo experimental La Posta, la cual pertenece a la Red Nacional de Estaciones 
Agroclimatológicas Automatizadas del INIFAP. Datos de precipitación pluvial no estuvieron disponibles para el periodo de estudio.

Las variables de respuesta estudiadas fueron número de garrapatas semi-repletas por bovino, ganancia de peso individual y ganancia de peso promedio por animal. El número de garrapatas semi-repletas por bovino consistió en el número de estos parásitos presentes en el lado izquierdo del animal en un periodo de 28 días. González-Cerón et al ${ }^{(6)}$ demostraron que esta variable tuvo una correlación alta (mayor a 0.90 ) con el número de garrapatas presentes en todo el cuerpo del animal. El número de garrapatas varió de 0 a 264, con un promedio general de 39.4 garrapatas por animal. A partir del número de garrapatas semi-repletas por bovino se determinó el número promedio de garrapatas por animal, el cual se utilizó para clasificar a los bovinos en: 1) bajamente infestados (0 a 30 garrapatas), 2) medianamente infestados (31 a 60 garrapatas) y 3) altamente infestados (61 garrapatas o más). La ganancia de peso individual consistió en los kilogramos de peso corporal ganados por el animal en un periodo de 28 días. La ganancia de peso promedio por animal se calculó dividiendo la suma de las ganancias de peso individuales obtenidas cada 28 días entre el número de pesajes.

El número de garrapatas semi-repletas por bovino se analizó con el procedimiento GENMOD (PROC GENMOD) del paquete estadístico $\mathrm{SAS}^{(7)}$, utilizando un modelo de mediciones repetidas que incluyó los efectos fijos de mes (periodo) de muestreo y sexo y grupo racial del becerro. Para la variable número de garrapatas se declaró una distribución Poisson como subrutina en el procedimiento GENMOD de $\mathrm{SAS}^{(7)}$; además, se aplicó una estructura de covarianzas auto-regresiva de primer orden $[\mathrm{AR}(1)]$. La ganancia de peso individual se analizó con el procedimiento MIXED (PROC MIXED) del programa SAS ${ }^{(7)}$, utilizando un modelo de mediciones repetidas que incluyó sexo y grupo racial del becerro como variables categóricas, y número de garrapatas semi-repletas por bovino, temperatura ambiental y humedad relativa como covariables, aplicando la misma estructura de covarianzas mencionada previamente. Para ganancia de peso individual se incluyeron en el modelo estadístico temperatura ambiental y humedad relativa en lugar de mes (periodo) de muestreo, porque no se tuvo interés en saber si existían diferencias entre meses en la ganancia de peso, sino que el objetivo fue ajustar de una manera más específica por factores climatológicos, para obtener el coeficiente de regresión lineal de la ganancia de peso individual sobre el número de garrapatas semi-repletas por bovino. La ganancia de peso promedio se analizó con el procedimiento GLM (PROC GLM) de SAS ${ }^{(7)}$, utilizando un modelo que incluyó nivel de infestación (alto, medio y bajo). En un análisis preliminar se encontró que los efectos sexo y grupo racial del becerro no fueron importantes $(P>0.05)$, por lo cual no se incluyeron en el modelo definitivo. En los análisis estadísticos del número de garrapatas semi-repletas por bovino y la ganancia de peso promedio, las diferencias entre medias se probaron con la opción PDIFF del programa SAS ${ }^{(7)}$. El grado de asociación entre número de garrapatas semirepletas por bovino con temperatura ambiental y humedad relativa, así como el grado de asociación entre el número promedio de garrapatas y la ganancia de peso promedio, se 
estimaron a través del coeficiente de correlación de Pearson, con el procedimiento CORR (PROC CORR) del paquete $\mathrm{SAS}^{(7)}$.

El promedio general de las ganancias de peso individuales fue $11.9 \mathrm{~kg}$. El número promedio de garrapatas semi-repletas, el nivel de infestación y la ganancia de peso promedio, por animal, se presentan en el Cuadro 1.

Cuadro 1: Número promedio de garrapatas (Boophilus microplus) semi-repletas (NPG), nivel de infestación (NIVEL) y ganancia de peso promedio (GPP; kg), por animal

\begin{tabular}{cclllllr}
\hline Animal & NPG & Nivel & GPP & Animal & NPG & Nivel & GPP \\
\hline 1 & 27 & Bajo & 12 & 17 & 87 & Alto & 3 \\
2 & 47 & Medio & 15 & 18 & 48 & Medio & 12 \\
3 & 45 & Medio & 12 & 19 & 58 & Medio & 12 \\
4 & 94 & Alto & 9 & 20 & 63 & Alto & 8 \\
5 & 30 & Bajo & 16 & 21 & 53 & Medio & 11 \\
6 & 45 & Medio & 13 & 22 & 65 & Alto & 12 \\
7 & 15 & Bajo & 13 & 23 & 50 & Medio & 12 \\
8 & 62 & Alto & 10 & 24 & 72 & Alto & 12 \\
9 & 25 & Bajo & 13 & 25 & 22 & Bajo & 15 \\
10 & 14 & Bajo & 15 & 26 & 36 & Medio & 14 \\
11 & 6 & Bajo & 14 & 27 & 65 & Alto & 10 \\
12 & 6 & Bajo & 13 & 28 & 39 & Medio & 11 \\
13 & 33 & Medio & 12 & 29 & 64 & Alto & 10 \\
14 & 32 & Medio & 18 & 30 & 62 & Alto & 10 \\
15 & 62 & Alto & 13 & 31 & 48 & Medio & 11 \\
16 & 15 & Bajo & 13 & & & & \\
\hline
\end{tabular}

El mes de muestreo resultó ser una fuente de variación significativa $(P=0.0199)$ para el número de garrapatas, el efecto lineal del número de garrapatas fue significativo $(P=0.0212)$ para ganancia de peso individual, y nivel de infestación fue significativo $(P=0.001)$ para ganancia de peso promedio. En julio, mes en que se presentó la mayor temperatura ambiental, el número promedio de garrapatas por animal fue mayor $(\mathrm{P}<0.05)$ que en los otros once meses del año (Cuadro 2). 
Cuadro 2: Medias de cuadrados mínimos con sus errores estándar para número de garrapatas (Boophilus microplus) semi-repletas, y medias de temperatura ambiental y humedad relativa, por mes

\begin{tabular}{lccc}
\hline Mes $^{\mathfrak{e}}$ & Número de garrapatas & $\begin{array}{c}\text { Temperatura } \\
\text { ambiental }\end{array}$ & $\begin{array}{c}\text { Humedad } \\
\text { relativa }\end{array}$ \\
\hline Enero & $20.7 \pm 2.5^{\mathrm{ef}}$ & 18.6 & 86.9 \\
Febrero & $18.5 \pm 2.7^{\mathrm{ef}}$ & 19.0 & 86.1 \\
Marzo & $32.4 \pm 4.5^{\mathrm{cd}}$ & 20.8 & 88.0 \\
Abril & $45.0 \pm 6.8^{\mathrm{b}}$ & 25.2 & 86.2 \\
Mayo & $43.0 \pm 4.4^{\mathrm{b}}$ & 26.2 & 85.6 \\
Junio & $45.1 \pm 4.3^{\mathrm{b}}$ & 25.9 & 85.4 \\
Julio & $62.9 \pm 6.9^{\mathrm{a}}$ & 26.8 & 87.7 \\
Agosto & $39.3 \pm 7.5^{\mathrm{bc}}$ & 26.0 & 87.0 \\
Agosto2 & $27.9 \pm 3.2^{\mathrm{cd}}$ & 26.5 & 87.8 \\
Septiembre & $24.5 \pm 2.9^{\mathrm{de}}$ & 25.1 & 91.0 \\
Septiembre2 & $16.3 \pm 3.3^{\mathrm{f}}$ & 25.8 & 89.9 \\
Octubre & $14.4 \pm 3.2^{\mathrm{f}}$ & 24.4 & 87.5 \\
Octubre2 & $5.9 \pm 1.6^{\mathrm{g}}$ & 25.2 & 87.3 \\
Noviembre & $32.5 \pm 3.4^{\mathrm{c}}$ & 21.5 & 86.0 \\
Diciembre & $17.4 \pm 2.5^{\mathrm{f}}$ & 20.6 & 89.4 \\
\hline
\end{tabular}

${ }^{£}$ Agosto2, Septiembre2 y Octubre2 se refieren a los meses de agosto, septiembre y octubre de 2015. ${ }_{\mathrm{a}, \mathrm{b}, \mathrm{c}, \mathrm{d}, \mathrm{e}, \mathrm{f}, \mathrm{g}}$ Medias con distinta literal son diferentes $(P<0.05)$.

Por ejemplo, en los casos más extremos, en el mes de julio los animales presentaron 46.6 y 57.0 garrapatas más que en los meses de septiembre (año 2015) y octubre (año 2015). En los meses de abril, mayo y junio, meses en los que la temperatura ambiental y la humedad relativa fueron similares, el número de garrapatas por animal también fue similar $(P>0.05), 45.0,43.0$ y 45.1 garrapatas, respectivamente. En enero y febrero, meses con una temperatura ambiental relativamente baja, los animales presentaron, en promedio, un número similar $(P>0.05)$ de garrapatas (20.7 y 18.5 , respectivamente); sin embargo, en estos dos primeros meses del año el número promedio de garrapatas por animal fue menor $(P<0.05)$ que en abril, mayo y junio, meses en los que se presentó una temperatura ambiental relativamente más alta. Estos resultados son acordes con el coeficiente de correlación de Pearson correspondiente, que indicó que a mayor temperatura ambiental mayor número de garrapatas en el animal (0.21; $P<0.0001)$; por el contrario, a menor humedad relativa mayor número de garrapatas en el animal $(-0.19 ; P<0.0001)$; sin embargo, la correlación entre este último par de características fue un poco más débil. Fluctuaciones en la población de garrapatas a lo largo del año también han sido observadas en reportes previos, donde la variación en la población dependió de las condiciones climáticas de cada región. Alonso-Díaz et al ${ }^{(8)}$, en Martínez de la Torre, Veracruz, encontraron que la mayor infestación por Rhipicephalus microplus en bovinos 3/4 Bos taurus $\mathrm{x} 1 / 4$ Bos indicus se presentó en los meses de mayo y junio (93 y 82 garrapatas, en 
promedio, por bovino), mientras que la menor infestación se presentó en noviembre y marzo ( $<10$ garrapatas, en promedio, por bovino), meses en los que la humedad relativa fue menor en comparación con los meses de mayo y junio.

En otra investigación un poco más reciente ${ }^{(9)}$, realizada con bovinos Criollo Lechero Tropical, en la zona centro del estado de Veracruz, se encontró que los meses de mayor infestación fueron agosto y octubre, con $11.1 \pm 0.6$ y $12.0 \pm 0.6$ garrapatas (Amblyomma cajennense + Boophilus microplus) por bovino, respectivamente; contrariamente, la menor infestación se presentó en mayo (2.9 \pm 0.6 garrapatas por animal), mes en que la precipitación pluvial fue más baja, en comparación con los meses de agosto y octubre (1.4 vs 11.9 y 17.9 $\mathrm{mm}$ ); sin embargo, en estos tres meses las temperaturas ambientales fueron similares. Otra investigación ${ }^{(10)}$ realizada en el trópico seco mexicano (Culiacán, Sinaloa), también reportó elevadas infestaciones en bovinos, principalmente durante los meses de julio a octubre, con 50 garrapatas en promedio por bovino, observándose una disminución durante los meses de noviembre y diciembre, con 30 garrapatas en promedio por bovino, disminución que coincidió con la época invernal, la cual se caracteriza por bajas temperaturas. Por su parte, Rodríguez-Gallegos y Acosta-Rodríguez ${ }^{(11)}$, en un estudio realizado con becerros de cruza terminal, encontraron un mayor número de garrapatas Boophilus microplus en febrero y marzo que en septiembre y octubre (155 \pm 10 vs $26 \pm 15)$, observándose que en los primeros meses del año la temperatura ambiental y la precipitación pluvial fueron menores que en los últimos meses del año (22.0 vs $26.4^{\circ} \mathrm{C}$, y 49 vs $\left.271 \mathrm{~mm}\right)$.

Los machos tendieron a tener más garrapatas que las hembras (29.9 vs 22.1 garrapatas; Cuadro 3); sin embargo, la diferencia no fue estadísticamente significativa $(P<0.055)$.

Cuadro 3: Medias de cuadrados mínimos con sus errores estándar e intervalos de confianza al $95 \%$ para número de garrapatas (Boophilus microplus) semi-repletas, por sexo

\section{Intervalo de confianza}

\begin{tabular}{lccc} 
Sexo & Media & Límite inferior & Límite superior \\
\hline Hembras & $22.1 \pm 2.3$ & 18.1 & 27.1 \\
Machos & $29.9 \pm 3.2$ & 24.2 & 37.0 \\
\hline
\end{tabular}

$(P>0.05)$.

En un estudio realizado en Australia ${ }^{(12)}$ se observó que el conteo de garrapatas fue $90 \%$ mayor en bovinos machos que en hembras, argumentando los autores que el efecto del sexo implica una fuerte influencia de las hormonas sexuales sobre la resistencia a dicho parásito. Por el contrario, Rodríguez-Gallegos y Acosta-Rodríguez ${ }^{(11)}$, utilizando animales de ocho meses de edad en condiciones de trópico húmedo, encontraron que becerros machos tuvieron niveles de infestación similares a los de becerros hembras. En un estudio realizado en México 
con la raza Criollo Lechero Tropical, González-Cerón et $a l^{(9)}$ tampoco encontraron diferencia en el número de garrapatas presentes en toretes y vaquillas (3.2 \pm 0.6 vs $3.5 \pm 0.4)$.

Los becerros 11/16 Suizo Pardo x 5/16 Cebú tendieron a tener menor número de garrapatas que los becerros de los otros grupos raciales, pero las diferencias entre los grupos raciales evaluados no fueron estadísticamente importantes $(P>0.05$; Cuadro 4$)$.

Cuadro 4: Medias de cuadrados mínimos con sus errores estándar e intervalos de confianza al 95\% para número de garrapatas (Boophilus microplus) semi-repletas, por grupo racial

\begin{tabular}{lcccc}
\hline \multirow{2}{*}{ Grupo racial $^{\mathfrak{t}}$} & \multicolumn{2}{c}{ Media } & \multicolumn{2}{c}{ Intervalo de confianza } \\
\cline { 3 - 4 } & & & Límite inferior & Límite superior \\
\hline $11 / 16 \mathrm{HO}$ & $28.7 \pm 2.1^{\mathrm{a}}$ & 24.9 & 33.0 \\
$11 / 16 \mathrm{SP}$ & $15.2 \pm$ & $0.4^{\mathrm{a}}$ & 14.5 & 16.0 \\
$3 / 4 \mathrm{HO}$ & $48.4 \pm 3.2^{\mathrm{a}}$ & 42.4 & 55.2 \\
$3 / 4 \mathrm{SP}$ & $37.3 \pm 5.6^{\mathrm{a}}$ & 27.8 & 50.0 \\
$5 / 8 \mathrm{HO}$ & $20.7 \pm 3.3^{\mathrm{a}}$ & 15.1 & 28.3 \\
$5 / 8 \mathrm{SP}$ & $30.8 \pm 18.4^{\mathrm{a}}$ & 9.5 & 99.6 \\
X HO & $34.6 \pm 5.5^{\mathrm{a}}$ & 25.4 & 47.2 \\
X SP & $32.4 \pm 2.4^{\mathrm{a}}$ & 28.0 & 37.4 \\
\hline
\end{tabular}

${ }^{£} 11 / 16 \mathrm{HO}=11 / 16$ Holstein $\times 5 / 16$ Cebú, 11/16 SP= 11/16 Suizo Pardo x 5/16 Cebú, 3/4 HO=3/4 Holstein $x$ 1/4 Cebú, 3/4 SP=3/4 Suizo Pardo x 1/4 Cebú, 5/8 HO= 5/8 Holstein x 3/8 Cebú, 5/8 SP= 5/8 Suizo Pardo x $3 / 8$ Cebú, $\mathrm{X}$ HO= Cruzas Holstein $\times$ Cebú indefinidas, $\mathrm{X} \mathrm{SP}=$ Cruzas Suizo Pardo $\mathrm{x}$ Cebú indefinidas. $(P>0.05)$.

Por el contrario, Alonso-Díaz et al ${ }^{(8)}$ encontraron que bovinos 3/4 B. taurus $\mathrm{x} 1 / 4 \mathrm{~B}$. indicus tuvieron un mayor número de garrapatas en la mayoría de los meses del año (enero, febrero, abril, mayo, junio, julio, septiembre, octubre, diciembre) que bovinos $1 / 2$. taurus $\times 1 / 2 B$. indicus; en otras palabras, los animales con un mayor porcentaje de genes de raza europea presentaron una mayor infestación por el ácaro; sin embargo, el intervalo del porcentaje de raza europea fue menor en el presente estudio $(62.5$ a $75.0 \%)$ que en el estudio citado ${ }^{(8)}$, donde dicho intervalo fue de 50 a $75 \%$, lo que implica una mayor diversidad (diferencia) genética entre bovinos. A pesar de ello, es probable que la inclusión de un mayor número de animales en el presente estudio hubiera resultado en menores errores estándar de las medias, permitiendo a su vez detectar diferencias en el número de garrapatas entre grupos raciales. Sutherst ${ }^{(13)}$ y Castro $^{(14)}$ habían reportado previamente que bovinos de razas Cebú (B. indicus) son más resistentes a las garrapatas que bovinos de razas europeas (B. taurus). Otros investigadores ${ }^{(15)}$ encontraron que el conteo diario de hembras Boophilus microplus repletas fue 3.3, 25.2, 22.5, 21.0 y 59.7 en bovinos Nelore, $1 / 2$ Nelore x $1 / 2$ Fleckvieh, $1 / 2$ Nelore x $1 / 2$ Chianina, $1 / 2$ Nelore $x 1 / 2$ Charolais y $3 / 8$ Nelore $x 5 / 8$ Angus. En condiciones de campo (infestación natural) en Australia ${ }^{(12)}$ se demostró que bovinos B. taurus $\mathrm{x}$ B. indicus portaron 
menos garrapatas que bovinos B. taurus (Shorthorn x Hereford). En un estudio realizado en Sudáfrica $^{(16)}$ se encontró que el número promedio de garrapatas fue 37.4, 24.1 y 5.3 en bovinos Hereford, Bonsmara (5/8 Afrikaner y 3/8 Hereford o Shorthorn) y Nguni (raza africana tropicalmente adaptada, producto de la combinación de razas B. taurus y B. indicus), respectivamente. Al respecto, se ha argumentado que el comportamiento etológico para evitar las garrapatas, la sensibilidad de la piel y un mayor acicalamiento en las razas Cebú pueden explicar la menor carga de este parásito comparada con la carga parasitaria en las razas exóticas $B$. taurus $^{(17)}$. Diferencias raciales en el número de garrapatas también han sido encontradas dentro de la especie indicus $^{(18)}$ donde se menciona que bovinos Brahman portaron dos veces más garrapatas (Boophilus microplus) que bovinos Nelore (animales de un año de edad en ambas razas). Por su parte, Rodríguez-Gallegos y Acosta-Rodríguez ${ }^{(11)}$ también encontraron efecto del grupo racial sobre el número de garrapatas, aunque no compararon animales $B$. taurus contra animales $B$. indicus y/o B. taurus x $B$. indicus, observando que becerros 1/2 Braunvieh-1/4 Holstein-1/4 Cebú tuvieron una mayor infestación natural que becerros 1/2 Angus Negro-1/4 Holstein-1/4 Cebú y 1/2 Angus Rojo-1/4 Holstein-1/4 Cebú.

El coeficiente de regresión lineal de la ganancia de peso sobre el número de garrapatas fue $0.03442 \mathrm{~kg} /$ garrapata $(P<0.05)$, lo que significa que la ganancia de peso disminuyó $34 \mathrm{~g}$ por cada garrapata semi-repleta presente en el animal en un periodo de 28 días. Por lo tanto, bajo las condiciones del presente estudio, en el mes de julio los animales perdieron, en promedio, $2.1 \mathrm{~kg}$ de peso corporal. En un estudio realizado con toretes Brahman, Brahman x Simmental, Sanga y Hereford, se estimó que la pérdida en la ganancia de peso fue de $28 \mathrm{~g}$ por garrapata (Amblyomma hebraeum) adulta repleta ${ }^{(19)}$, resultado similar al encontrado en el presente estudio. Por el contrario, Frisch y O’Neill ${ }^{(20)}$ reportaron que el coeficiente de regresión de la ganancia de peso sobre el número de garrapatas fue $-0.42 \mathrm{~kg} /$ garrapata $/ 10$ meses, lo que significa que la ganancia de peso en dicho estudio disminuyó 420 g por garrapata en un periodo de 10 meses. También se ha reportado que cada garrapata repleta (Rhipicephalus appendiculatus) estuvo asociada con una pérdida de peso corporal de $4.4 \mathrm{~g}$, pérdida menor a la encontrada en la presente investigación ${ }^{(21)}$.

El coeficiente de correlación de Pearson $(-0.67 ; P<0.0001)$ reveló que la ganancia de peso promedio fue menor conforme aumentó el número de garrapatas en los animales. Similarmente, en un estudio realizado en Texas con bovinos Angus x Cebú ${ }^{(22)}$ se estimó una correlación de -0.61 entre la ganancia de peso y el número acumulado de garrapatas (Amblyomma americanum). En otro estudio realizado en Zambia con bovinos de la raza Sanga $^{(23)}$ se encontró que la ganancia de peso estuvo negativa y moderadamente correlacionada con el número de garrapatas (Amblyomma variegatum) en dos diferentes hatos (-0.72 y -0.70), resultado similar al encontrado en el presente estudio. En otro estudio realizado en Camerún ${ }^{(24)}$ con bovinos de la raza Gudali (B. indicus), también se encontró una correlación negativa y moderada entre la ganancia de peso y el número de garrapatas 
(Amblyomma variegatum); sin embargo, en este último estudio la correlación fue menor $(-0.52)$.

Los bovinos con niveles medios y bajos de infestación ganaron, en promedio, 2.88 y $3.87 \mathrm{~kg}$ más $(P<0.05)$ por periodo de muestreo, respectivamente, que aquellos con altos niveles de infestación (Cuadro 5).

Cuadro 5: Medias de cuadrados mínimos con sus errores estándar e intervalos de confianza al 95\% para ganancia de peso promedio $(\mathrm{kg})$, por nivel de infestación

\begin{tabular}{lrcc}
\hline \multirow{2}{*}{ Nivel de infestación } & Media & \multicolumn{2}{c}{ Intervalo de confianza } \\
\cline { 3 - 4 } & & Límite inferior & Límite superior \\
\hline Alto & $9.82 \pm 0.66^{\mathrm{a}}$ & 8.46 & 11.18 \\
Medio & $12.70 \pm 0.61^{\mathrm{b}}$ & 11.46 & 13.94 \\
Bajo & $13.69 \pm 0.70^{\mathrm{b}}$ & 12.25 & 15.12 \\
\hline
\end{tabular}

Estos resultados son parcialmente similares a los obtenidos en Brasil con ganado HolsteinCebú ${ }^{(25)}$ donde se observó que animales con bajos niveles de infestación tuvieron mayor ganancia de peso que animales con niveles medios y altos de infestación; sin embargo, este estudio no describe como se realizó la categorización de los animales con base en el número de garrapatas que portaban los mismos. Similarmente, Corrier et $\mathrm{l}^{(26)}$, al evaluar la carga de garrapatas repletas (Boophilus microplus; diámetro $>5 \mathrm{~mm}$ ) en bovinos de la raza Normando, encontraron que animales altamente infestados (138 a 300 garrapatas) pesaron $24 \mathrm{~kg}$ menos en el día 125 de la prueba que animales ligeramente infestados ( 0 a 33 garrapatas). Varios autores han reportado que existen animales que consistentemente portan menos garrapatas que otros en el mismo ambiente, debido a su mayor habilidad para responder inmunológicamente a las garrapatas ${ }^{(27-29)}$.

En conclusión, la infestación por el ácaro Boophilus microplus requiere un control más estricto en los meses de mayor temperatura ambiental (abril a julio). El sexo y el genotipo de los bovinos no influyeron en la cantidad de garrapatas que portaron los animales. La correlación entre el número promedio de garrapatas y la ganancia de peso promedio fue negativa y moderada, encontrándose que bovinos con altos niveles de infestación con este vector tuvieron menores ganancias de peso que bovinos con medios y bajos niveles de infestación. 


\section{Literatura citada:}

1. González SPJR, Hernández OR. Boophilus microplus: estado actual de la resistencia a los acaricidas en la frontera México Estados Unidos y su impacto en la relación comercial. Rev Mex Cienc Pecu 2012;3(Supl 1):1-8.

2. Rajput ZI, Song-hua H, Wan-jun Ch, Arijo AG, Chen-wen X. Importance of ticks and their chemical and immunological control in livestock. J Zhejiang Univ Science B 2006;7(11):912-921.

3. García BA. Situación actual de la campaña nacional contra la garrapata en México. IV Seminario Internacional de Parasitología Animal. Puerto Vallarta, Jalisco, México. 1999:47-50.

4. Wharton RH, Utech KBW. The relation between engorgement and dropping of $B$. microplus (Canestrini) (Ixodidae) to the assessment of tick numbers on cattle. J Aust Entomol Soc 1970;9:171-182.

5. Rodríguez-Vivas RI, Domínguez AJ, Cob GL. Técnicas diagnósticas de parasitología veterinaria. Mérida, Yucatán, México. Universidad Autónoma de Yucatán. 1994:131140 .

6. González-Cerón F, Becerril-Pérez CM, Torres-Hernández G, Díaz-Rivera P. Garrapatas que infestan regiones corporales del bovino Criollo Lechero Tropical en Veracruz, México. Agrociencia 2009;43(1):11-19.

7. SAS Institute Inc. SAS/STAT® 9.3 User's guide. Cary, NC: SAS Institute Inc. 2011.

8. Alonso-Díaz MA, López-Silva BJ, de Magalhães-Labarthe ACL, Rodríguez-Vivas RI. Infestación natural de hembras de Boophilus microplus Canestrini, 1887 (Acari: Ixodidae) en dos genotipos de bovinos en el trópico húmedo de Veracruz, México. Vet Méx 2007;38(4):503-509.

9. González-Cerón F, Becerril-Pérez CM, Torres-Hernández G, Díaz-Rivera P, SantellanoEstrada E, Rosendo-Ponce A. Infestación natural por Amblyomma cajennese y Boophilus microplus en bovinos Criollo Lechero Tropical durante la época de lluvias. Agrociencia 2009;43:577-584.

10. Gaxiola CSM, Borbolla IJE, Quintero MMT, Rodríguez MJ, Borbolla IJ, Castro CN, et al. Infestación natural de bovinos con Boophilus microplus en el municipio de Culiacán, Sinaloa, México [resumen]. IV Seminario Internacional de Parasitología Animal. Puerto Vallarta, Jalisco, México. 1999:225-226.

11. Rodríguez-Gallegos CE, Acosta-Rodríguez MR. Genetic and environmental factors influencing the resistance of terminal cross calves to tick Rhipicephalus (Boophilus) 
microplus and horn fly Haematobia irritans. Trop Subtrop Agroecosys 2011;13:437444.

12. Seifert GW. Variations between and within breeds of cattle in resistance to field infestations of the cattle tick (Boophilus microplus). Aust J Agric Res 1971;22:159-168.

13. Sutherst RW. Resistance of cattle to ticks and one element in control programme in Mexico. FAO Animal Production and Health Paper. 1989; Pp. 154-164.

14. Castro JJ. Sustainable tick and tickborne disease control in livestock improvement in developing countries. Vet Parasitol 1997;71:77-97.

15. Gomes A, Honer MR, Schenck MAM, Curvo JBE. Populations of the cattle tick Boophilus microplus on purebred Nelore, Ibagé and NelorexEuropean crossbreds in the Brazilian Savanna. Trop Anim Health Prod 1989;21:20-24.

16. Scholtz MM, Spickett AM, Lombard PE, Enslin CB. The effect of tick infestation on the productivity of cows of three breeds of cattle. Onderstepoort J Vet Res 1991;58:71-74.

17. Meltzer MI. A possible explanation of the apparent breed-related resistance in cattle to Bont tick (Amblyomma hebraeum) infestations. Vet Parasitol 1996;67:275-279.

18. Brizuela CM, Ortellano CA, Sanchez TI, Walker AR. Formulation of an integrated control of Boophilus microplus in Paraguay. Vet Parasitol 1996;63:95-108.

19. Norval RAI, Sutherst RW, Kerr JD. Infestations of the bont tick Amblyomma hebraeum (Acari: Ixodidae) on different breeds of cattle in Zimbabwe. Exp Appl Acarology 1996;20:599-605.

20. Frisch JE, O'Neill CJ. Comparative evaluation of beef cattle breeds of African, European and Indian origins. 2. Resistance to cattle ticks and gastrointestinal nematodes. Anim Sci 1998;67:39-48.

21. Norval RAI, Sutherst RW, Kurki J, Gibson JD, Kerr JD. The effect of the brown ear-tick (Rhipicephalus appendiculatus) on the growth of Sanga and European breed cattle. Vet Parasitol 1988;30:149-164.

22. Tolleson DR, Teel PD, Stuth JW, Strey OF, Welsh Jr. TH, Carstens GE, et al. Effects of a lone star tick (Amblyomma americanum) burden on performance and metabolic indicators in growing beef steers. Vet Parasitol 2010;173:99-106.

23. Pegram RG, Lemche J, Chizyuka HGB, Sutherst RW, Floyd RB, Kerr JD, et al. Effect of tick control on liveweight gain of cattle in central Zambia. Med Vet Entomol 1989;3:313-320. 
24. Stachurski F, Musonge EN, Achu-kwi MD, Saliki JT. Impact of natural infestation of Amblyomma variegatum on the liveweight gain of male Gudali cattle in Adamawa (Cameroon). Vet Parasitol 1993;9:299-311.

25. Labruna MB, Veríssimo CJ. Observações sobre a infestação por Boophilus microplus (Acari: Ixodidae) em bovinos mantidos em rotação de pastagem, sob alta densidade animal. Arq Inst Biol, São Paulo, 2001;68(2):115-120.

26. Corrier DE, Vizcaino O, Terry M, Betancourt A, Kuttler KL, Carson CA, et al. Mortality, weight loss and anaemia in Bos taurus calves exposed to Boophilus microplus ticks in the tropics of Colombia. Trop Anim Health Prod 1979;11:215-221.

27. Mattioli RC, Pandey VS, Murray M, Fitzpatrick JL. Immunogenetic influences on tick resistance in African cattle with particular reference to trypanotolerant N'Dama (Bos taurus) and trypanosusceptible Gobra zebu (Bos indicus) cattle. Acta Tropica 2000;75:263-277.

28. Das G, Ghosh S, Ray DD. Reduction of Theileria annulata infection in ticks fed on calves immunized with purified larval antigens of Hyalomma anatolicum anatolicum. Trop Anim Health Prod 2005;37:345-361.

29. Jonsson NN. The productivity effects of cattle tick (Boophilus microplus) infestation on cattle, with particular reference to Bos indicus cattle and their crosses. Vet Parasitol 2006;137:1-10. 